\title{
Brownian motion in a classical ideal gas: A microscopic approach to Langevin's equation
}

\author{
RANGAN LAHIRI $^{1, *}$, ARVIND $^{2,3}$ and ANIRBAN SAIN ${ }^{4,5}$ \\ ${ }^{1}$ Department of Physics, Indian Institute of Science, Bangalore 560 012, India \\ ${ }^{2}$ Department of Physics, Carnegie Mellon University, Pittsburgh, PA 15213, USA \\ ${ }^{3}$ Permanent address: Department of Physics, Guru Nanak Dev University, \\ Amritsar 143 005, India \\ ${ }^{4}$ Department of Physics, University of Waterloo, Ontario, Canada N2L3G1, Canada \\ ${ }^{5}$ Present address: Department of Physics, Indian Institute of Technology, Powai, \\ Mumbai 400 076, India \\ E-mail: xarvind@andrew.cmu.edu; asain@phy.iitb.ac.in
}

MS received 3 February 2003; revised 12 January 2004; accepted 17 January 2004

\begin{abstract}
We present an insightful 'derivation' of the Langevin equation and the fluctuation dissipation theorem in the specific context of a heavier particle moving through an ideal gas of much lighter particles. The Newton's law of motion $(m \ddot{x}=F)$ for the heavy particle reduces to a Langevin equation (valid on a coarser time-scale) with the assumption that the lighter gas particles follow a Boltzmann velocity distribution. Starting from the kinematics of the random collisions we show that (1) the average force $\langle F\rangle \propto-\dot{x}$ and (2) the correlation function of the fluctuating force $\eta=F-\langle F\rangle$ is related to the strength of the average force.
\end{abstract}

Keywords. Brownian motion; fluctuation dissipation theorem; ideal gas.

PACS Nos 05.40.-a; 05.40.Jc; 05.20.Dd

\section{Introduction}

In recent years, many experiments on single large molecules like colloidal spheres and bio-polymers, have been reported [1]. Many of these experiments focus on the kinematics of motion of these large molecules through typical fluid environments, at room temperature. Most theoretical explanations of these experiments $[1,2]$ neglect the intrinsic correlations of the surrounding fluid and treat the fluid as a bath,

${ }^{*}$ Deceased 
generating friction and uncorrelated noise on the large molecule, exactly the way a Brownian particle is treated.

This approach may fail if the molecule is large or if it is close to a hard wall such that the displaced fluid due to its motion does not return to equilibrium immediately. In that case one may have to solve the Navier-Stokes equation to correctly incorporate the dynamics of the fluid, for example while deriving the Stokes' law [3]. Further, extended macromolecules of the polymer variety, where one part of the molecule disturbs the fluid which in turn affects the other parts of the molecule (the so-called hydrodynamic effect [4]) require yet another kind of analysis. It is however worthwhile and desirable to gain more physical insight and understanding into the simple yet intriguing situation of the Brownian motion, for its own sake and also to come up with meaningful models for more complicated situations. We revisit the problem of Brownian motion through an ideal gas in this work.

When a particle, substantially heavier than the gas particles, is injected into the gas, it is randomly kicked around by the gas molecules. However, due to the heavier nature of this particle (usually called the Brownian particle) it takes a bunch of small kicks by the gas molecules, over a time interval, to move the Brownian particle appreciably. Thus there occurs a natural separation of time-scales for the motion of this heavier particle and the gas molecules [5,6]. The motion of a Brownian particle, on a coarse grained time-scale is usually described by the Langevin equation $[6,7]$,

$$
M \ddot{x}+\Gamma \dot{x}=\eta,
$$

where $x$ is the position of the Brownian particle. The overall effect of collisions is modeled by introducing the damping coefficient $\Gamma$ and a random force $\eta$ with zero mean. This is a physically motivated phenomenological equation where the effect of collisions is neatly separated into velocity-dependent friction and a random force, with a non-trivial relationship between the two via the fluctuation dissipation theorem (FDT) [8].

In the general context of many-body systems, the Langevin equation provides a description of the slow degrees of freedom, while treating the fast modes as noise acting on the slower ones. Apart from Brownian motion, thermally activated barrier crossing phenomena [9], polymer dynamics in a solvent fluid [4] and epitaxial growth of a surface profile due to random deposition [10] are a few examples of such a situation and the description assumes that a distinct natural separation of timescales exists between the fast and the slow degrees of freedom. The non-triviality of the Langevin description lies in splitting the effect of the fast degrees of freedom into a systematic part (friction) and a random noise obeying FDT. Mostly, the Langevin equation is motivated from a phenomenological point of view. Every derivation of the Langevin equation requires certain assumptions; Mori and Zwanzig have given a formal prescription (projection operator method [11]) to derive the Langevin equation starting from a microscopic picture. Given a Langevin equation, Kubo's method $[12,13]$ can be used to derive FDT with certain assumptions. Assuming the motion of the Brownian particle through an ideal gas to be a jump Markov process, Gillespie [14] and van Kampen [15] have derived the Langevin equation. A more physical approach to derive Langevin equation and FDT, though in one dimension, has also been reported [16]. 


\section{Brownian motion in a classical ideal gas}

In this paper we present a new approach to derive the Langevin equation for a Brownian particle moving in an ideal gas at a fixed temperature, using a combination of microscopic and statistical ideas. The work we present here differs from others mainly in the way we derive the FDT and that we consider two different limits of the ideal gas, namely, small mean free path and large mean free path.

The fact that for a moving Brownian particle the relative velocity of the gas particles approaching it from the front is higher than the ones approaching from behind, causes retardation leading to net damping. So the collisional force, though random in nature, has a finite average $\langle F\rangle(=-\Gamma \dot{x}$, in eq. (1)). If we separate out this time-independent average from the total force, what remains (i.e., $F-\langle F\rangle$ ) is a time varying random force $\eta$. For the Langevin description to be valid the probability distribution of $\eta$ must satisfy FDT.

We explicitly show this for two different limits of the ideal gas, namely the one with high collision rate and the other with negligible collision rate.

This paper is arranged as follows: In $\S 2$ we calculate the damping constant and derive the fluctuation dissipation theorem for a gas with a low collision rate. The gas molecules in this case do not suffer too many collisions among themselves in the time taken by the Brownian particle to gain a sufficient amount of momentum. Section 3 deals with similar questions for an ideal gas with a high collision rate and in this case the gas particles can equilibrate in a short time compared to the time taken by the Brownian particle to gain momentum. Section 4 contains a discussion of the results and some conclusions. In Appendix A we discuss the case when the Brownian particle is assumed to be a sphere instead of a disk and Appendix B has some useful mathematical formulae.

\section{Brownian motion in an ideal gas with mean free path $\lambda \rightarrow \infty$}

Consider a Brownian particle $B$ moving in a gas along the $x$ direction with a velocity $u_{0}$. The Brownian particle is assumed to be substantially heavier than a gas particle and therefore it moves at much slower speeds at comparable kinetic energies. To find the friction coefficient $\Gamma$, we have to calculate the net force on $B$ due to collisions with the gas molecules. Consider the simplest possible geometry for $B$, that of a disc moving with a velocity perpendicular to its plane (in Appendix $\mathrm{A}$, the calculation is extended to a spherical particle). It is easier to capture the essentials of the calculation in this case without getting lost in the geometrical factors. The easiest way to arrive at the force is to treat all collisions in the rest frame of the Brownian particle. In this frame if a molecule comes in with a velocity $\mathbf{v}=v_{x} \hat{x}+v_{y} \hat{y}+v_{z} \hat{z}$ then for a disc, heavy and large compared to the gas molecules, it follows from the conservation of energy and momentum that it is reflected with a velocity $\mathbf{v}^{\prime}=-\kappa v_{x} \hat{x}+v_{y} \hat{y}+v_{z} \hat{z}$ where

$$
\kappa=\frac{M-m}{M+m},
$$

$M$ and $m$ being the masses of the Brownian particle and the gas molecule respectively. For $M \gg m$, the momentum transferred to $B$ per collision is then

$$
\delta \mathbf{p}=(1+\kappa) m v_{x} \hat{x} .
$$




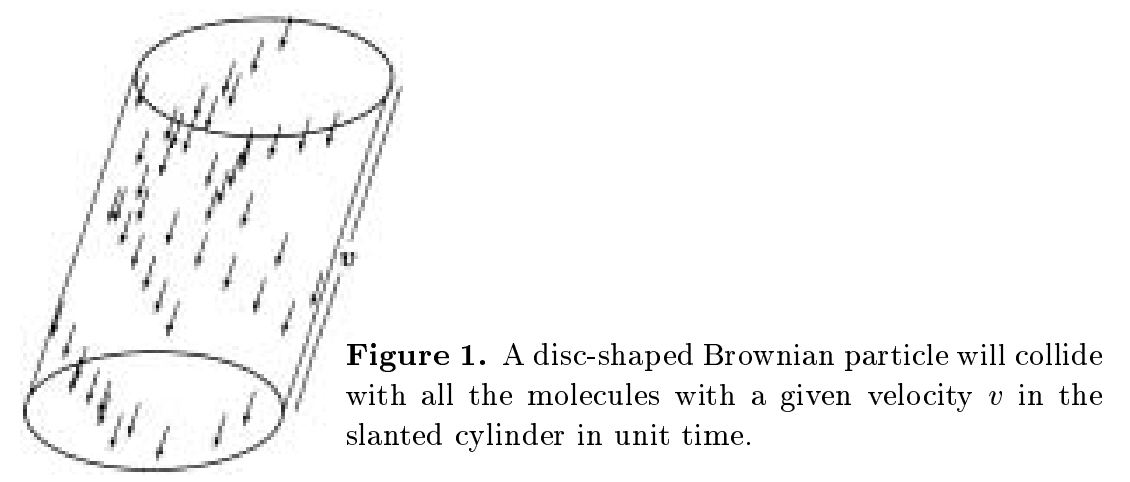

The force on $B$ is the momentum transferred to it per unit time, for which we need to know the number of collisions per unit time for each value of $\mathbf{v}$. This number for a given $\mathbf{v}$ is the number of molecules in the (slanted) cylinder of edge length $|\mathbf{v}|$ swept in unit time as shown in figure 2., i.e.

$$
N_{\mathrm{col}}=A \frac{\rho}{m}\left|v_{x}\right|,
$$

where $A$ is the area of cross-section of the plate and $\rho$ the density of gas. The force on $B$ is $N_{\text {col }}$ times the momentum transfer in each collision, integrated over all possible velocities:

$$
F_{x}=-A \rho(1+\kappa) \int \mathrm{d} \mathbf{v} P_{c}(\mathbf{v})\left|v_{x}\right| v_{x},
$$

where $P_{c}(\mathbf{v})$ is the distribution of velocities in the frame of the Brownian particle. Since the gas is at equilibrium and it has uniform density, the Boltzmann transport equation gives the steady state solution to be a Maxwellian [17,18], i.e.,

$$
\begin{aligned}
& P\left(v_{x}, v_{y}, v_{z}\right)=P_{0}\left(v_{x}\right) P_{0}\left(v_{y}\right) P_{0}\left(v_{z}\right) \\
& P_{0}\left(v_{x}\right)=\frac{1}{\sqrt{2 \pi} \sigma} \mathrm{e}^{-v_{x}^{2} / 2 \sigma^{2}}
\end{aligned}
$$

where $\sigma$ is the mean thermal velocity component in one direction.

$$
\sigma^{2}=\left\langle v_{x}^{2}\right\rangle_{\mathrm{th}}=\frac{k_{\mathrm{B}} T}{m} .
$$

If the velocity of a gas molecule is $\mathbf{v}$ in the co-moving frame, then it is $\mathbf{v}+u_{0} \hat{\mathbf{x}}$ in the lab frame. Therefore the distribution in the co-moving frame is,

$$
P_{c}\left(v_{x}, v_{y}, v_{z}\right)=P_{0}\left(v_{x}+u_{0}\right) P_{0}\left(v_{y}\right) P_{0}\left(v_{z}\right) .
$$

There is no component of the force perpendicular to $\hat{x}$ whereas the $x$ component is

$$
\begin{aligned}
F_{x} & =A \rho(1+\kappa) \int_{-\infty}^{\infty} \mathrm{d} v_{x} P_{0}\left(v_{x}+u_{0}\right)\left|v_{x}\right| v_{x} \\
& =A \rho(1+\kappa) \int_{-\infty}^{\infty} \mathrm{d} v_{x} P_{0}\left(v_{x}\right)\left|v_{x}-u_{0}\right|\left(v_{x}-u_{0}\right) .
\end{aligned}
$$




\section{Brownian motion in a classical ideal gas}

After a few simple manipulations, we get

$$
F_{x}=-2 A \rho(1+\kappa) \mathrm{e}^{-u_{0}^{2} / 2 \sigma^{2}} \int_{0}^{\infty} \mathrm{d} v_{x} v_{x}^{2} \frac{1}{\sqrt{2 \pi}} \mathrm{e}^{-v_{x}^{2} / 2 \sigma^{2}} \sinh \left(\frac{v_{x} u_{0}}{\sigma^{2}}\right) .
$$

It may be noted that the force is an odd function of $u_{0}$, in keeping with the general rule that dissipative terms in an equation of motion violate time reversal invariance. For the velocities $u_{0}$ small compared to the average thermal velocities in the gas, we can Taylor-expand to the lowest order in the dimensionless parameter $u_{0} / \sigma$ to get

$$
F_{x}=-2 A \rho(1+\kappa) u_{0} \sigma \frac{1}{\sqrt{2 \pi}} \int_{0}^{\infty} \mathrm{d} x x^{3} \mathrm{e}^{-x^{2} / 2} .
$$

We call the dimensionless integral in the above equation as $\chi_{3}$ (Appendix B). The final expression for the force then is

$$
F_{x}=-2 A \rho(1+\kappa) u_{0} \sigma \chi_{3}
$$

from which we read off the friction coefficient

$$
\Gamma=2 A \rho(1+\kappa) \sigma \chi_{3} .
$$

The friction coefficient is not proportional to the linear dimension of the particle (as it is in the Stokes' law) but rather to the area. This result carries over to the case of the sphere as well. The area comes because the gas we are considering is ideal and the motion of the Brownian particle in no way affects the flow of the gas. The Stokes' result essentially derives from the fact that the fluid (being a strongly interacting gas) makes way for the particle as it moves through it [3].

Let us now proceed with the calculation of the noise. In the standard Langevin equation (1) the noise $\eta$ has to satisfy the fluctuation dissipation theorem

$$
\langle\eta(t) \eta(\hat{t})\rangle=2 k_{\mathrm{B}} T \Gamma \delta(t-\hat{t}) .
$$

This theorem relies on the requirement that the Brownian particle is in thermal equilibrium with the gas it is moving through.

Before we derive the fluctuation dissipation theorem, we coarse grain the time in terms of a small time unit $\epsilon$, chosen such that several collisions take place within $\epsilon$ time, while the Brownian particle does not move appreciably during this interval. For a massive enough particle it is always possible to find such a time. It is simplest to calculate the noise in the rest frame of the particle, where the discretized version of the Langevin equation (1) is

$$
M \frac{\Delta v}{\epsilon}=\eta
$$

The desirable fluctuation dissipation theorem that the noise is expected to satisfy then reads in discrete form as

$$
\left\langle\eta_{i} \eta_{j}\right\rangle=2 k_{\mathrm{B}} T \Gamma \delta_{i j} / \epsilon .
$$


Note, that the function $\delta\left(t_{i}-t_{j}\right) \rightarrow \delta_{i j} / \epsilon$, such that for $i=j, \delta(0) \rightarrow 1 / \epsilon$, which is the appropriate discrete form [19]. This is the relation we will now derive from the kinematics of the collisions between the Brownian particle and the gas molecules. The momentum along $x$, transferred to $B$ in the interval $\epsilon$ is $\epsilon \eta$, which we call $\delta p_{x}$. We have to show that

- The momentum transferred $\delta p_{x}$ is distributed independently in each time interval $\epsilon$.

- The mean square value of $\delta p_{x}$ in any given time is given by

$$
\left\langle\left(\delta p_{x}\right)^{2}\right\rangle=2 k_{\mathrm{B}} T \Gamma \epsilon .
$$

The first of the above statements follows from the fact that the gas is ideal, so that each collision is independent and the coarse graining over time will obviously not generate correlations. This corresponds to the linearized Boltzmann transport equation [18]. Suppose a total of $N$ collisions take place in the interval $\epsilon$, counting all velocities. The total momentum transferred is [20]

$$
\delta p_{x}=m(1+\kappa)\left(v_{x 1}+\cdots+v_{x N}\right) .
$$

Once again, since the velocities are independent, all cross terms have a zero average, leading to

$$
\left\langle\left(\delta p_{x}\right)^{2}\right\rangle=m^{2}(1+\kappa)^{2} N\left\langle v_{x}{ }^{2}\right\rangle .
$$

The number of particles of velocity $\mathbf{v}$ that collide in time $\epsilon$ is

$$
N(\mathbf{v})=\frac{\rho}{m} A \epsilon P_{0}(\mathbf{v})\left|v_{x}\right| .
$$

The total number of collisions is most easily calculated in Cartesian coordinates:

$$
\begin{aligned}
N & =A \frac{\rho}{m} \epsilon \int \mathrm{d} \mathbf{v} P_{c}(\mathbf{v})\left|v_{x}\right| \\
& =2 A \frac{\rho}{m} \sigma \epsilon \chi_{1}+\mathcal{O}\left(\left(u_{0} / \sigma\right)^{2}\right) .
\end{aligned}
$$

A calculation in polar coordinates to the leading order gives

$$
N=A \frac{\rho}{m} \sigma \epsilon \chi_{3}
$$

which is the same result as $\chi_{3} / \chi_{1}=2$ (as shown in Appendix B). The average $\left\langle v_{x}^{2}\right\rangle$ is not the thermal average, but the average over the sample of particles that collide with $B$ in the interval $\epsilon$.

$$
\begin{aligned}
\left\langle v_{x}^{2}\right\rangle & =\frac{\int \mathrm{d} \mathbf{v} N(\mathbf{v}) v_{x}^{2}}{\int \mathrm{d} \mathbf{v} N(\mathbf{v})}=\frac{1}{N} A \frac{\rho}{m} \epsilon \int \mathrm{d} \mathbf{v} P_{c}(\mathbf{v}) v_{x}^{2}\left|v_{x}\right| \\
& =\frac{1}{2 \sigma \chi_{1}} 2 \sigma^{3} \chi_{3}+\mathcal{O}\left(\left(u_{0} / \sigma\right)^{2}\right) \\
& \simeq 2 \sigma^{2} .
\end{aligned}
$$



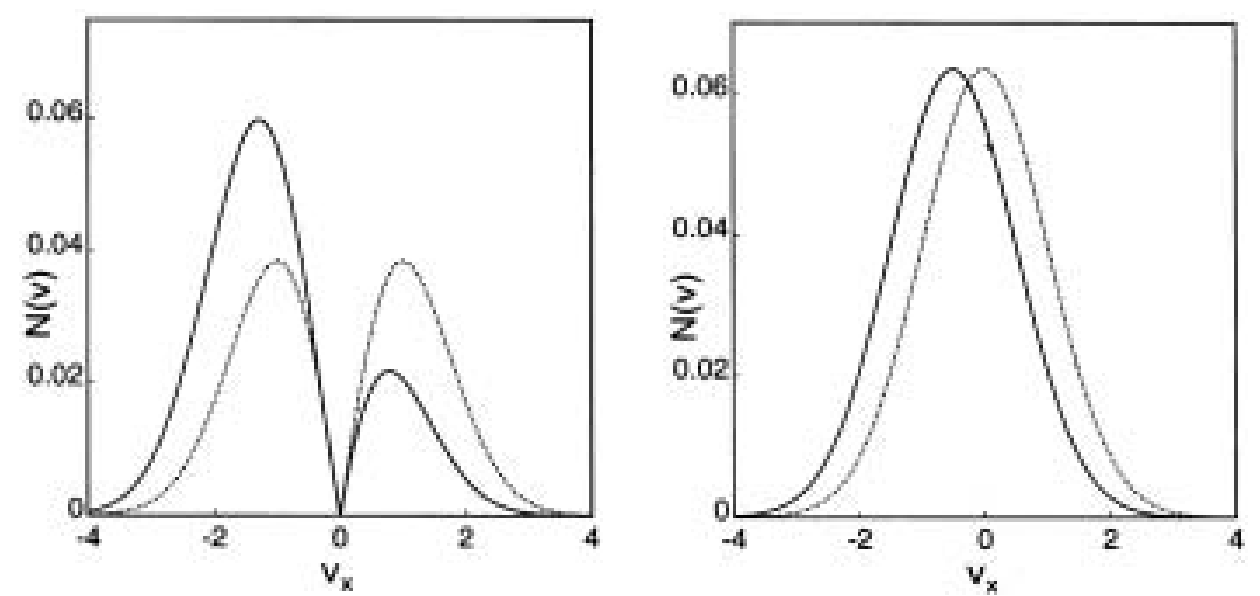

Figure 2. Plot of the distribution $N(v)$ for the ideal gas in two different limits. The left plot is for an ideal gas with large mean free path while the right one is for a gas with very small mean free path. The dotted curves in both the cases are for a Brownian particle at rest and the solid lines for a moving Brownian particle.

Note that the mean square velocity in eq. (23) is larger than the thermal average velocity $\sigma^{2}$. This is due to the fact that in the ensemble of molecules that collide in a given interval, there are a greater number of faster molecules, as they sweep a larger cylinder (figure 2). Substituting for $N$ and $\left\langle v_{x}^{2}\right\rangle$ in eq. (19) we get

$$
\left\langle\left(\delta p_{x}\right)^{2}\right\rangle=m^{2}(1+\kappa)^{2} 2 A \frac{\rho}{m} \epsilon \sigma \chi_{1} 2 \sigma^{2} .
$$

Combining eqs (24) and (13) and using eqs (7) and (B2) the above equation becomes

$$
\left\langle\left(\delta p_{x}\right)^{2}\right\rangle=(1+\kappa) k_{\mathrm{B}} T \Gamma \epsilon
$$

which for $\kappa=1$, i.e., for $M \gg m$ goes to the desired fluctuation dissipation result (17). Also note that the change of momentum (or velocity) of the Brownian particle, in a time step $\epsilon$, is of the order $\sqrt{\epsilon}$ and not $\epsilon$, which might be naively expected. This is an essential feature of Langevin equation [19].

\section{Brownian motion in an ideal gas with small mean free path}

The classical ideal gas is a familiar concept, described by the equation of state

$$
P V=n R T .
$$

This equation of state is a limiting case of a more physically reasonable equation of state, namely the real gas (Van der Waals gas) equation [17]

$$
\left(P-\frac{a}{V^{2}}\right)(V-b)=n R T
$$


where $a$ and $b$ represent the intermolecular forces and the volume occupied by the gas molecules respectively.

The meaning of working in the ideal gas limit corresponds to neglecting all forces between the particles other than the two-body hard sphere collisions, and neglecting the volume occupied by the molecules themselves as compared to the volume of the gas. Assume that we have $N$ gas molecules of diameter $\sigma$ moving in a volume $V$. The volume occupied by the gas molecules is proportional to $N \sigma^{3}$ and one knows from kinetic theory that the mean free path is proportional to $V / N \sigma^{2}$. The ideal gas limit corresponds to $\left(N \sigma^{3} / V\right) \rightarrow 0$. It turns out that there is no unique way of reaching such a limit and different limiting procedures lead to different physical situations.

One way is to consider a very large mean free path $\left(V / N \sigma^{2}\right) \rightarrow \infty$ which automatically leads to the ideal gas limit $\left(N \sigma^{3} / V\right) \rightarrow 0$. In this case, as a consequence of dilution, the collision rate is reduced and finally goes to zero while the mean free path approaches infinity. Though such a gas will satisfy the ideal gas equation of state, it will have infinite relaxation time, and will never equilibrate on its own! However, once the equilibrium is established it is maintained in the absence of external perturbations. This is the kind of gas we considered for the passage of a Brownian particle in $\S 2$. However, this is an overkill. In order to achieve the limit $\left(N \sigma^{3} / V\right) \rightarrow 0$ we need not go to the infinite mean free path limit. Keeping in mind that $N$ is large $\left(\sim 10^{23}\right)$ and $\sigma$ is very small in comparison to $V^{1 / 3}$ we can achieve very small values of $N \sigma^{3}$ without making $N \sigma^{2}$ small. This second way is more interesting, in that one can consistently carry out the limiting process from a real gas to an ideal gas while retaining a finite collision rate. In fact it is possible to fix the collision rate at any desired value and go through the limiting procedure. As a consequence we can make the relaxation time as small as we wish. Therefore, if disturbed from equilibrium, such a gas will equilibrate on its own [21]. Both these cases correspond to different physical situations and the behaviour of a Brownian particle injected in either of the gases is different in each case.

Going back to the motion of a Brownian particle in the second type of gas we observe that the relaxation time (the time in which the gas goes back to equilibrium, after being disturbed) can be made smaller than the coarse graining time (the time for which the Brownian particle does not change its velocity appreciably). In fact we are allowed to assume any pre-assigned value for the relaxation time and other parameters can be adjusted accordingly. The picture of the previous case where we assumed that the gas is practically collisionless (at least within coarse graining time $\epsilon$ ) is no longer valid. The number of gas particles colliding with the Brownian particle per unit time at velocity $\mathbf{v}$ is no longer the number of particles in the slanted cylinder of height $v_{x}$, as the particles keep colliding and forgetting their velocities within the time $\epsilon$.

It turns out that it is simpler to compute the collision rate in this case as we do not have to worry about the slanted cylinder. The gas particles colliding with the Brownian particle are chosen randomly from a continuously equilibrating velocity distribution, as seen from the rest frame of the Brownian particle. The memory effect present in the slanted cylinder description of the previous section disappears. The number of collisions per unit time $N(\mathbf{v})$ of the gas molecules moving with a velocity $\mathbf{v}$ with the Brownian particle in this case (figure 2 ) is 


$$
N(\mathbf{v})=K \frac{\rho}{m} A v_{\mathrm{th}} P_{c}(\mathbf{v}),
$$

where $v_{\text {th }}=\sqrt{k_{\mathrm{B}} T / m}$ is the thermal velocity, $K$ is a proportionality constant defined through (31) and all other parameters have the same meaning as in eq. (20). The average force can now be readily obtained as in the previous case

$$
F=\int N(\mathbf{v}) \delta \mathbf{p} \mathrm{d} \mathbf{v} .
$$

Using the value of $\delta \mathbf{p}$ from eq. (3) and of $N(\mathbf{v})$ from eq. (28) we have

$$
F=K(1+\kappa) \rho A v_{\text {th }} \int P_{c}(\mathbf{v}) \mathbf{v} \mathrm{d} \mathbf{v}=-u_{0} \Gamma,
$$

where the friction coefficient $\Gamma=K(1+\kappa) \rho A v_{\text {th }}$. It is interesting that the friction being proportional to the velocity of the Brownian particle is an exact result here.

We now move on to the noise calculation, the treatment being very similar to that of $\S 2$. The momentum transferred in each time $\epsilon$ is obviously independent and we basically only have to show that $\left\langle p^{2}\right\rangle=2 k_{\mathrm{B}} T \Gamma \epsilon$. Equation (19) is still valid for the Brownian plate where only the $x$ component of momentum is important but the expressions for the total number of collisions $N$ and $\left\langle v_{x}^{2}\right\rangle$ are different in this case. We have for the Brownian particle at rest

$$
N=\int N(\mathbf{v}) \mathrm{d} \mathbf{v}=K \frac{\rho}{m} A v_{\text {th }}
$$

and since there are no memory effects we have the pure thermal velocity distribution with

$$
\left\langle v_{x}^{2}\right\rangle=\frac{k_{\mathrm{B}} T}{m} .
$$

Using these expressions and eq. (19) we get

$$
\left\langle p_{x}^{2}\right\rangle=m^{2}(1+\kappa)^{2} K \frac{\rho}{m} A v_{\mathrm{th}} \epsilon \frac{k_{\mathrm{B}} T}{m}=(1+\kappa) \epsilon k_{\mathrm{B}} T \Gamma .
$$

In the limit when Brownian particle is of large mass compared to the gas molecules, i.e., $\kappa \rightarrow 1$, we recover the fluctuation dissipation theorem (17). The derivation has been simpler and more exact in this case, where the gas is constantly equilibrating. We have not used any approximations here as opposed to $\S 2$. It is explicitly clear in this case (though it is true for both the ensembles) that the separation of time-scales for the motion of the gas molecules and that of the Brownian particle is essential for the fluctuation dissipation theorem to hold and thus the theorem is valid in its form given in eq. (14) in the limit of $\kappa \rightarrow 1$.

\section{Concluding remarks}

We have shown, how the familiar result of Langevin equation and fluctuation dissipation theorem can be derived and understood in a special case when the fluid 
through which the Brownian particle moves is an ideal gas. The frictional force comes about because of the fact that if $B$ moves in a particular direction then as seen from the frame of $B$ the velocity of gas molecules hitting it from the front is more compared to that for the molecules hitting it from behind. As the details of the calculations in $\S \S 2$ and 3 show, the situation is a little more subtle and we have to be careful while setting up the expression for the collision rate as a function of velocity. Moreover, the separation of time-scales for the motion of the Brownian particle and the gas particles is essential for obtaining the fluctuation dissipation theorem.

We observe that the essential difference in the two kinds of ideal gases is in the distribution $N(\mathbf{v})$ of collision rate at velocity $\mathbf{v}$. For the ideal gas of the first kind, this function tends to have larger values for larger velocities as compared to the Maxwellian, as the volume of the cylinder from which all the molecules must collide with $B$ is directly proportional to $|\mathbf{v}|$. On the other hand, for the ideal gas of the second type, the absence of memory effects and the breakdown of the cylinder picture prevents any such biases.

Another interesting observation is that the friction coefficient is proportional to the cross-sectional area for the cases of both the plate and the sphere. This is unlike the case of Stokes' law where it is proportional to the radius. This is due to the fact that we have neglected the back reaction due to the motion of the sphere on the velocity distribution of the gas particles, which has been retained to be Maxwellian all along. On the contrary, for the calculation of the Stokes' law, one takes into account the effect of the motion of the sphere on the fluid velocity. While our treatment holds for a dilute non-interacting gas, the Stokes' law is obeyed in fluids with higher density. In Stoke's law regime, one considers the passage of a macroscopic particle through an incompressible fluid which is treated in the continuum approximation. The movement creates a gap and hence a negative pressure behind the particle which then pulls the particle backwards leading to the viscous drag. The size of the moving object is therefore assumed to be bigger than the gas particles as well as the mean free path. In our calculations, we work with a Brownian particle which is bigger than the gas particles, but smaller than the mean free path. It is not truly a macroscopic object and we are not working with an incompressible fluid. To get an idea about the orders of magnitudes involved, consider the following example. The diameter of nitrogen/oxygen molecule is roughly $4 \times 10^{-8} \mathrm{~cm}$. The mean free path for air at room temperature and atmospheric pressure is of the order of $7 \times 10^{-6} \mathrm{~cm}$ (if one reduces the pressure to 1 Torr the mean free path becomes as large as $5 \times 10^{-3} \mathrm{~cm}$ ). This allows for a variety of choices of objects which are larger and much heavier than the gas molecules and are still very much smaller than the mean free path. Nanoparticles of size between $10^{-7} \mathrm{~cm}$ and $10^{-5}$ $\mathrm{cm}(1-100 \mathrm{~nm})$ being generated in laboratories these days can be used for such an experiment. If a beam of such particles is injected into air at room temperature then at an appropriate pressure one should be able to observe a friction coefficient proportional to area as opposed to the Stoke's law. Another candidate for a Brownian particle can be a large heavy molecule which satisfies the conditions we need. However the experiment might be difficult to do in terms of controlling the number of particles injected and to ensure that the particles do not settle too quickly under gravity. 
We feel that our analysis can be carried out for other kinds of Brownian motion as well. One could, for example, consider the Brownian motion of angular variables for molecules moving in an ideal gas. We expect that interesting physical insights can be gained if such an analysis is carried out. A similar analysis should also be possible for the classical experiment of observing Brownian motion of the angular degree of freedom of a mirror suspended in a gas at low pressure and the rotational fluctuations seen by reflecting a light beam from the mirror (suggested by the referee).

\section{Appendix A: Calculation of friction coefficient for a Brownian sphere}

In this appendix, we demonstrate that the friction coefficient can be calculated for the case when the Brownian particle is a sphere instead of a plate. The underlying physics is same though the calculation is a little more involved. The plate was chosen only for simplicity and this calculation is given here to convince the reader that there is nothing special about the plate. We will see that the only difference from the plate case is a geometrical factor.

Imagine the Brownian particle to be a sphere of radius $R$ moving in the $\hat{\mathbf{z}}$ direction with velocity $u_{0}$ (as shown in figure 3 ). We consider a frame of reference $F$ which is at rest with respect to the Brownian particle. Consider the collision of the Brownian particle with a beam of particles moving with velocity $\mathbf{V}\left(V_{0}, \theta^{\prime}, \phi^{\prime}\right)$ in this frame. Further, consider a frame $F^{\prime}$ again at rest with respect to the Brownian particle but with its orientation such that the $\hat{\mathbf{z}}^{\prime}$-axis is in the direction of $\mathbf{V}$. When a narrow beam hits the sphere at a point $(R, \theta, \phi)$ in the frame $F^{\prime}$, it is reflected by the sphere and the force exerted on the sphere, which is along $\mathbf{z}^{\prime}$ direction is given by

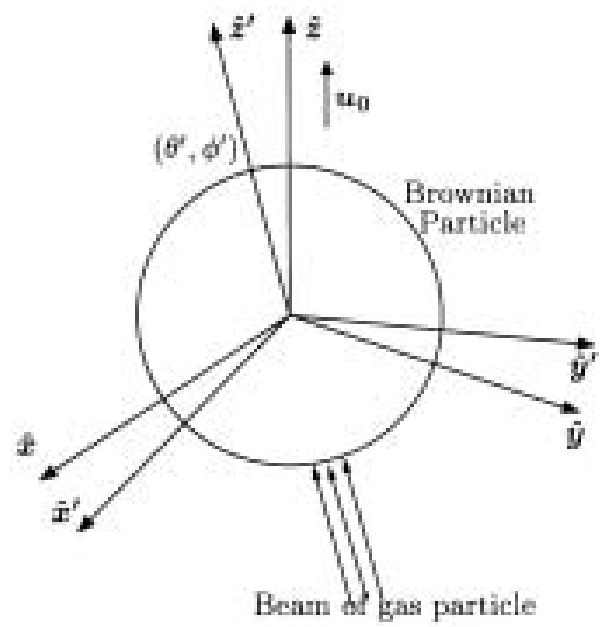

Figure 3. A pictorial description of collisions for a spherical Brownian particle. 


$$
\mathbf{F}_{\hat{\mathbf{z}}^{\prime} \text {-rest }}=\text { flux } \cdot(1+\kappa) \cdot V_{0} \cos \theta \hat{\mathbf{z}}^{\prime},
$$

where

$$
\text { flux }=\rho \mathbf{V} \cdot(-\mathrm{d} \mathbf{s})=\rho V_{0} \cos \theta R^{2} \sin \theta \mathrm{d} \theta \mathrm{d} \phi .
$$

The velocity distribution of eq. (8) breaks the $\theta^{\prime}$ symmetry but preserves the $\phi^{\prime}$ symmetry. Therefore in the frame $F$ the $\hat{\mathbf{x}}$ and $\hat{\mathbf{y}}$ component of the force averages to zero and we need to consider only the $\hat{\mathbf{z}}$ component of the force.

$$
F_{\hat{\mathbf{z}}}=F_{\hat{\mathbf{z}}^{\prime} \text {-rest }} \cdot \cos \theta^{\prime} .
$$

Rewriting eq. (8) for the velocity distribution in the spherical polar coordinates as seen from the frame $F$ we have

$$
P_{c}(\mathbf{V})=\frac{1}{(\sqrt{2 \pi} \sigma)^{3}} \mathrm{e}^{-\left[\left(V_{0} \cos \theta^{\prime}+u_{0}\right)^{2}+\left(V_{0} \sin \theta^{\prime}\right)^{2}\right] / 2 \sigma^{2}} .
$$

The total frictional force can now be readily obtained by integrating over all the angular variables and the velocity distribution

$$
\begin{aligned}
F_{\hat{\mathbf{z}}}= & C_{1} C_{2} \int_{0}^{\infty} \mathrm{d} V_{0} V_{0}^{4} \mathrm{e}^{-V_{0}^{2} / 2 \sigma^{2}} \\
& \times \int_{0}^{\pi} \frac{1}{(\sqrt{2 \pi} \sigma)^{3}} \mathrm{e}^{2 u_{0} V_{0} \cos \theta^{\prime} / 2 \sigma^{2}} \sin \theta^{\prime} \cos \theta^{\prime} \mathrm{d} \theta^{\prime}
\end{aligned}
$$

where

$$
C_{1}=(2 \pi)^{2}(1+\kappa) R^{2} \mathrm{e}^{-u_{0}^{2} / 2 \sigma^{2}}
$$

and

$$
C_{2}=\int_{0}^{\pi} \mathrm{d} \theta \sin \theta \cos ^{2} \theta=2 / 3 .
$$

To the lowest order, $\int \mathrm{d} \theta^{\prime}$ gives $u_{0} V_{0} / 3 \sigma^{2}$ and by working out $\int \mathrm{d} V_{0}$ we obtain the total frictional force

$$
F_{\hat{\mathbf{z}}}=-C_{1} C_{2} \frac{u_{0} \sigma}{6 \pi} \chi_{5} .
$$

Using the dimensionless integral $\chi_{5}$ from Appendix B, the relation $\chi_{5}=4 \chi_{3}$ and substituting the values of $C_{1}, C_{2}$, the final expression for the force simplifies to

$$
F_{\hat{\mathbf{z}}}=-\frac{8}{9}\left(2 A \rho(1+\kappa) u_{0} \sigma \chi_{3}\right),
$$

where $A=\pi R^{2}$ is the cross-section of the sphere. We notice that the only difference in this expression as compared to the plate case of eq. (12) is the geometrical factor of $8 / 9$. 


\section{Appendix B: Some useful integrals}

We list some useful integrals used in the paper.

$$
\begin{aligned}
& \Gamma(n)=\int_{0}^{\infty} \mathrm{d} x x^{n-1} \mathrm{e}^{-x}=2 \int_{0}^{\infty} \mathrm{d} x x^{2 n-1} \mathrm{e}^{-x^{2}} \\
& =2^{1-n} \int_{0}^{\infty} \mathrm{d} x x^{2 n-1} \mathrm{e}^{-x^{2} / 2} \\
& \chi_{n}=\frac{1}{\sqrt{2 \pi}} \int_{0}^{\infty} \mathrm{d} x x^{n} \mathrm{e}^{-x^{2} / 2}=\frac{1}{\sqrt{2 \pi}} 2^{(n-1) / 2} \Gamma\left(\frac{n+1}{2}\right) \\
& \chi_{3} / \chi_{1}=2, \quad \chi_{5} / \chi_{3}=4 .
\end{aligned}
$$

\section{Acknowledgements}

The authors thank Deepak Dhar and Supurna Sinha for useful comments and discussion.

\section{References}

[1] M A Bevan and D C Prieve, J. Chem. Phys. 113, 1228 (2000) J Meiners and S R Quake, Phys. Rev. Lett. 84, 5014 (2000)

[2] D S Sholl, M Fenwick, E S Atman and D C Prieve, J. Chem. Phys. 113, 9268 (2000)

[3] L D Landau and E M Lifshitz, Fluid mechanics, 2nd edition (Pergamon Press, Oxford, 1987)

[4] M Doi and S F Edwards, The theory of polymer dynamics (Oxford University Press, 1986) Ch. 3, Sec. 6

[5] A Einstein, Ann. Phys.(Leipzig) 17, 549 (1905); appearing in The collected papers of Albert Einstein, English translation by Anna Beck (Princeton University Press, 1989) vol. 2, p. 123

[6] P Langevin, Comptes. Rendues 146, 530 (1908); for English translation, see Don S Lemons and Anthony Gythiel, Paul Langevin's 1908 paper 'On the Theory of Brownian Motion', Am. J. Phys. 65, 1079 (1997)

[7] S Chandrasekhar, Rev. Mod. Phys. 15, 1 (1943)

[8] P M Chaikin and T Lubensky, Principles of condensed matter physics (Cambridge University Press, Cambridge, 1995)

[9] P Hanngi, P Talkner and M Borokovek, Rev. Mod. Phys. 62, 251 (1990)

[10] J Krug and H Spohn, Kinetic roughening of growing surfaces, in Solids far from equilibrium edited by C Godreche (Cambridge University Press, 1992) pp. 479-582

[11] H Grabert, Projection operator techniques in nonequilibrium statistical mechanics (Springer, Berlin, 1982)

[12] R Kubo, Rep. Prog. Phys. 29, 255 (1966)

[13] B U Felderhof, J. Phys. A: Math. Gen. 11, 921 (1978) 
[14] D T Gillespie, Am. J. Phys. 61, 1077 (1993); 64, 225 (1996)

D T Gillespie, Markov processes: an introduction for physical scientists (Academic Press, Boston, 1992)

[15] N G van Kampen, Stochastic processes in physics and chemistry (North Holland, New York, 1992)

[16] B G De Grooth, Am. J. Phys. 67, 1248 (1999)

[17] K Huang, Statistical mechanics (Wiley, New York, 1963)

[18] Carlo Cercignani, The Boltzmann equation and its applications (Springer-Verlag, New York, 1987).

[19] J Zinn-Justin, Quantum field theory and critical phenomena (Clarendon Press, Oxford, 1989) Ch. 3

[20] In fact the number of collisions in the interval is itself a fluctuating quantity, however, in our calculation we assume it to be a constant. This is a good approximation as long as $N$ is large, as the fluctuation in $N$ is $O(\sqrt{N})$, which is much smaller than $N$. Since $N$ is proportional to the size of the Brownian particle as well as to the interval $\epsilon$, it is always large for a large particle. Note that a more massive particle moves less in a given interval, so $\epsilon$ can be chosen to be large. Hence both the mass and the size of the particle contribute to increasing $N$.

[21] For a discussion, see [18], p. 43 